\title{
A measure of neurobehavioral functioning after coma. Part II: Clinical and scientific implementation
}

\author{
Theresa Louise-Bender Pape, DrPH, MA, CCC-SLP/L; $;^{1-4}$ Ricardo G. Senno, MD, MS; \\ Ann Guernon, MS, CCC-SLP/L; ${ }^{3}$ James P. Kelly, MA, MD $^{5}$ \\ ${ }^{1}$ Department of Veterans Affairs (VA), Veterans Health Administration, Research Service, Edward Hines Jr. VA Hospi- \\ tal, Hines, IL; ${ }^{2}$ Marianjoy Rehabilitation Hospital, Wheaton, IL; Northwestern University, Feinberg School of Medi- \\ cine, ${ }^{3}$ Department of Physical Medicine and Rehabilitation and ${ }^{4}$ Institute for Health Services Research and Policy \\ Studies, Chicago, IL; ${ }^{5}$ University of Colorado School of Medicine, Department of Neurosurgery, Denver, CO
}

\begin{abstract}
This longitudinal validation study is Part II of a twopart series. Part I focuses on the methods used to construct the neurobehavioral measure derived from the Disorders of Consciousness Scale (DOCS) as well as the evidence of reliability and validity. Part II illustrates, through a series of selected case reports, the clinical use of repeated DOCS measures to enhance and complement medical rehabilitation management. The use of repeated DOCS measures in scientific investigations of mechanisms of injury is also described. Participants included patients at rehabilitation hospitals who were 18 years of age and older and unconscious after severe brain injury. Medical decision making regarding short-term effects of pharmacological intervention was augmented and improved through the examination of individual neurobehavioral recovery patterns. We identified medications to treat secondary medical complications and successfully determined effective dosage, presumably improving prognosis for recovery. We facilitated and enhanced development and refinement of individualized rehabilitation programs. Two investigations of treatment effectiveness during coma recovery and examination of the relationship between behavioral changes and neural adaptation are also described. By systematically tracking and mapping individual patterns of neurobehavioral recovery, we show that medical and rehabilitation management after coma can be enhanced. In addition, we also show that by examining the relationship between the DOCS neurobehavioral measure with mechanistic indicators of neurological recovery such as functional magnetic resonance imaging, scientific investigations of treatment and rehabilitation effectiveness can be enhanced.
\end{abstract}

Key words: brain injury, coma recovery, measurement, medical management, outcomes, prognostication, translation of research into practice.

\section{INTRODUCTION}

The capacity for scientists to examine treatment effectiveness, for physicians to detect secondary medical complications, and for therapists to develop rehabilitation

Abbreviations: $\mathrm{BI}=$ brain injury, $\mathrm{BID}=$ bis in die or two times daily, CT = computed tomography, DOCS = Disorders of Consciousness Scale, EEG = electroencephalogram, GCS = Glasgow Coma Scale, IP = inpatient, PRN = pro re nata or as needed, $\mathrm{QD}=$ quaque die or one time daily, $\mathrm{QEEG}=$ quantitative electroencephalogram, QID = quater in die or four times daily, TBI = traumatic brain injury, TID = ter in die or three times daily, VA = Department of Veterans Affairs, VP = ventriculoperitoneal.

This material was based on work supported by the Department of Veterans Affairs (VA), Veterans Health Administration, Rehabilitation Research and Development Service, through career development grant B2632-V to Dr. Pape. One of the intervention studies described in this paper is made possible, in part, because of the support of the General Clinical Research Center (GCRC) at Northwestern University's Feinberg School of Medicine. The GCRC is supported in part by grant M01 RR-00048 from the National Center for Research Resources, National Institutes of Health.

*Address all correspondence to Dr. Theresa Louise-Bender Pape, Department of Veterans Affairs (VA), Veterans Health Administration, Research Service, Edward Hines Jr. VA Hospital, PO Box 5000 (M/C 151H), Hines, IL 60141; fax: 708-2027487; email: Theresa.Pape@med.va.gov

DOI: 10.1682/JRRD.2004.03.0033 
goals during coma recovery is limited because the unconscious participant is not able to report symptoms and/or actively participate in testing. Medical treatments to alleviate complications impeding recovery (e.g., spasticity, seizures) include pharmacological agents. Pharmacological agents are also used to facilitate neurobehavioral responsiveness and recovery. Pharmacological efforts to treat complications and low levels of responsiveness may, however, result in unintended sedation, which can be confused with lack of neurobehavioral recovery. Practitioners have not been able to determine whether pharmacological efforts work as intended because the capacity to reliably and accurately measure change in neurobehavioral functioning has not been available to them. A further challenge with unconscious persons is the development of acute rehabilitation goals and plans. Therapists are charged with the task of developing individualized rehabilitation plans, but establishing goals and tracking progress toward these goals are not possible without the capacity of the therapists to reliably and accurately detect and track neurobehavioral recovery. Part I, of this two-part series of papers, summarized the evidence regarding the reliability and validity of a neurobehavioral assessment tool-the Disorders of Consciousness Scale (DOCS). This paper, Part II of the series, presents four cases that illustrate how the mapping of neurobehavioral recovery patterns is being implemented clinically with the use of repeated measures derived from the DOCS and describes how these repeated measures are being used scientifically.

Rehabilitation medicine aims to facilitate the recovery of function after severe brain injury (BI). While medical management has practice parameters [1], insufficient scientific evidence exists to support evidence-based practice guidelines regarding medical and rehabilitation management [2-6]. Clinicians use neuropharmacological interventions such as methylphenidate, cholinergic agents, and serotonin reuptake inhibitors to control agitation and to improve attention and memory. Despite widespread offlabel use, evidence regarding the effectiveness of neuropharmacology is inconclusive [7-10]. Evidence of improved functioning given behavioral interventions, such as sensory stimulation, is also inconclusive [11]. The paucity of evidence has been due, in part, to the lack of reliable and valid methods that detect and track neurobehavioral functioning in unconscious persons over time.

In lieu of evidence-based practice guidelines, standard medical practices include obtaining medical history, monitoring vital signs and sleep/wake cycles, conducting informal observations, having therapists report their observations, completing neuroimaging electroencephalogram (EEG) studies, and monitoring intracranial pressure to establish baseline indicators for unconscious persons. If a deviation occurs from these baseline indicators, then it can signal the possibility of undetected secondary medical complications and/or improvement in health status, but this practice is considered to be unreliable $[2,6,12]$. Standard rehabilitation practice includes the development of individualized rehabilitation plans, and behavioral assessments are conducted to establish goals and to monitor progress [1]. Despite the lack of scientific evidence, sensory stimulation has been used as the standard rehabilitation intervention to achieve these goals during coma recovery [11].

\section{METHODS}

For the larger observational study described in Part I of this series (page 1), research procedures included mapping each subject's neurobehavioral results after two time points. The principal investigator routinely provided the results to each subject's family and rehabilitation team. As part of this routine research procedure, several cases highlight the usefulness of mapping the patterns of neurobehavioral change. We present four cases in this paper to illustrate how repeated measures derived from the DOCS can be used to complement medical decision making. The first author is also conducting two separate intervention studies of unconscious persons in which functional magnetic resonance imaging (fMRI) is used to examine the relationship between the recovery of function and neural adaptation in unconscious persons. In this paper, we describe the research methods for these studies to illustrate how repeated DOCS measures are being used to advance clinical research. The human subject institutional review boards (IRBs) at participating hospitals approved these studies.

\section{Data Collection Procedures}

Clinicians evaluated each subject weekly up to 6 weeks with the DOCS and independently classified him or her as being in a coma, a vegetative state (VS), a minimally conscious state (MCS), or conscious according to clinical criteria (see Part I: Theory, reliability, and validity of Disorders of Consciousness Scale, of this issue, Appendix, Table 2, available in online version only) 
[2,13-17]. After inpatient (IP) rehabilitation discharge, the researchers follow each subject monthly up to 1 year to identify when or if he or she recovers consciousness. The 1-year outcome interviews include more comprehensive data collection regarding functional outcomes.

\section{Neurobehavioral Mapping of Repeated DOCS Measures: Clinical Implementation}

We present the four cases in this section to illustrate how the DOCS neurobehavioral measure was used over time to compare and contrast the short-term effects of dantrolene and oral baclofen, to examine the short-term effects of a dopaminergic stimulant, and to illustrate how routine examinations of neurobehavioral recovery patterns helped determine effective antiseizure medications and doses as well as the development of individualized rehabilitation plans. The medical rehabilitation teams used the results to guide care during coma recovery.

Neurobehavioral functioning is mapped for each of the four cases in scatter plots and bar graphs (Figures 1-4). Higher DOCS measures indicate either higher levels of neurobehavioral functioning or higher levels of difficulty associated with a given test stimuli [18]. We illustrate each participant's neurobehavioral recovery pattern in these plots by contrasting the DOCS measure with days after injury that the DOCS evaluation was completed. Each participant's rehabilitation intensity is reported as the average hours of speech, physical and occupational therapy, and evaluations received per IP rehabilitation day (i.e., a 7-day week in which admission and discharge days are included in the denominator). Medications administered at times of DOCS evaluations are reported according to dose and frequency (i.e., four times daily or quater in die [QID], one

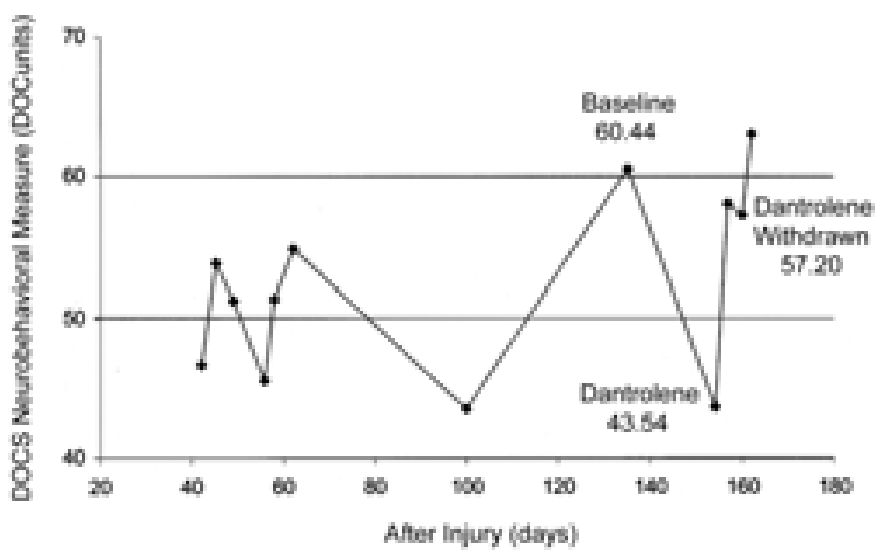

Figure 1.

Unmanageable spasticity. DOCS $=$ Disorders or Consciousness Scale. time daily or quaque die [QD], three times daily or ter in die [TID], two times daily or bis in die [BID], or as needed or pro re nata [PRN]).

\section{Case 1: Unmanageable Spasticity}

A 20-year-old female incurred a closed-head injury subsequent to a motor vehicle accident in which she was an unbelted passenger projected through the windshield. She presented in the emergency room with a Glasgow Coma Scale (GCS) score of 3 . She then suffered respiratory arrest with subsequent anoxic damage. Her acute medical hospitalization was complicated by liver, spleen, and kidney lacerations as well as splenectomy, bilateral upper-limb

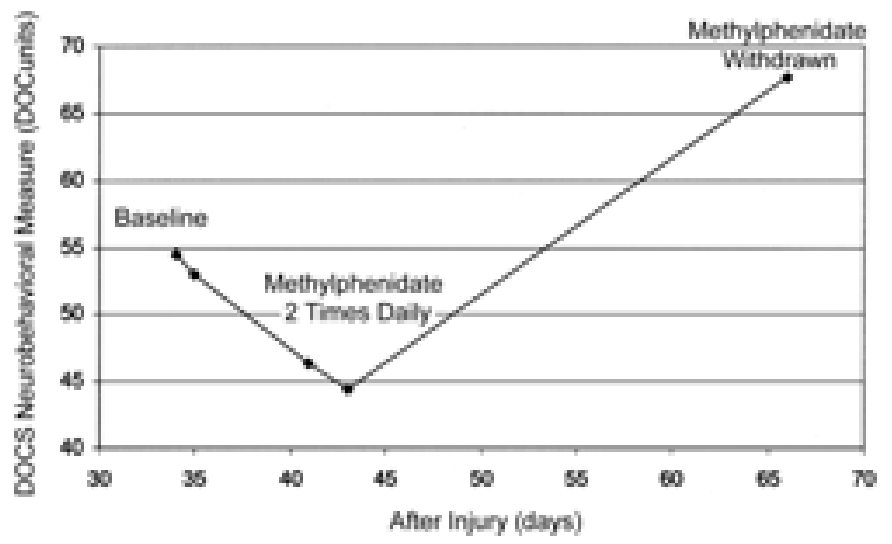

Figure 2.

Methylphenidate in an 18-year-old "adult." DOCS = Disorders of Consciousness Scale.

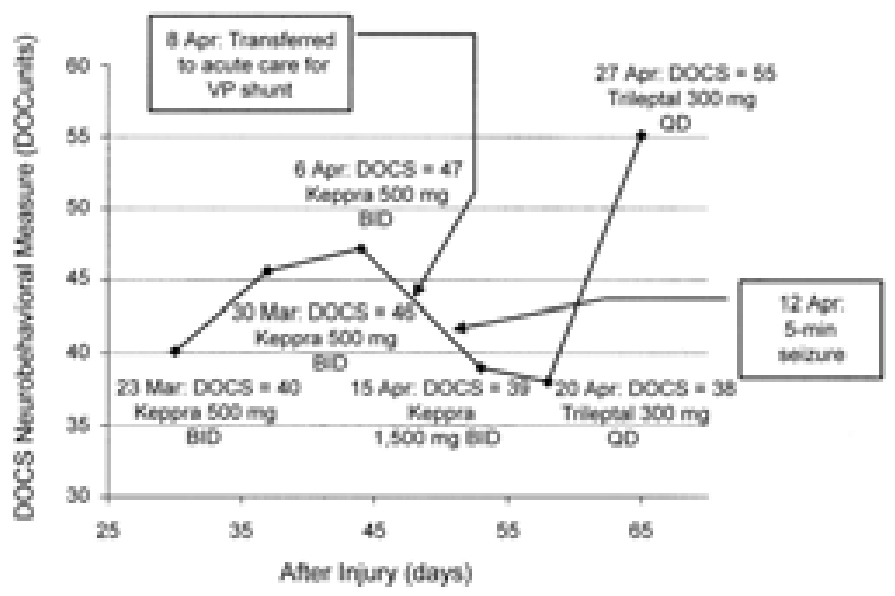

Figure 3.

Seizure activity. QID = two times daily, BID = one time daily, DOCS = Disorders of Consciousness Scale, and VP = ventriculoperitoneal. 
JRRD, Volume 42, Number 1, 2005

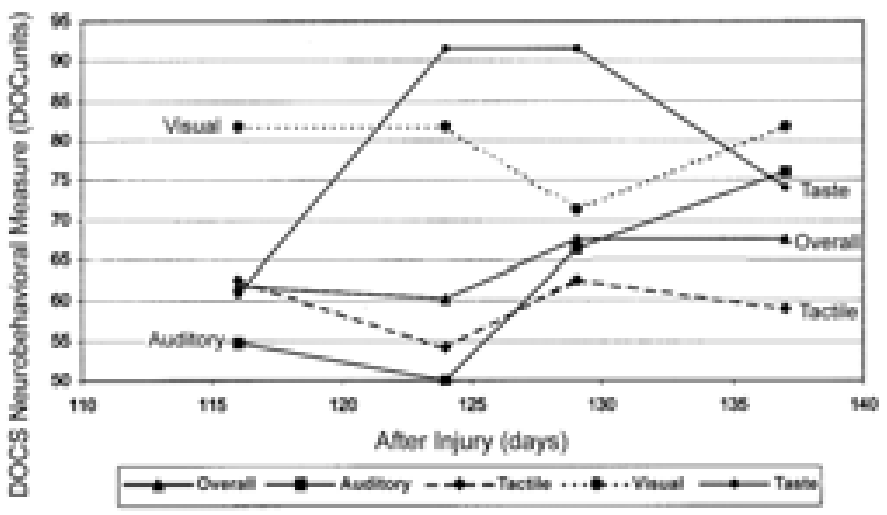

Figure 4.

Individualized rehabilitation goals: Development and refinement. DOCS $=$ Disorders of Consciousness Scale.

deep venus thrombosis, intracranial hemorrhage, right clavicular and left acetabular fracture, pneumonia, sepsis, respiratory arrest with ventilatory support, and right diaphragm repair. She was admitted to IP rehabilitation 42 days after injury and her rehabilitation stay was complicated by hyperadrenergicity and severe spasticity. She was discharged 69 days after injury and readmitted to IP rehabilitation 135 days after injury for spasticity management. She received 3.5 hours of therapy per IP rehabilitation day. The participant's physical therapists reported that dantrolene improved spasticity, but the family reported that the participant was not as active during therapy sessions and activities outside of therapy when given this medication. Our purpose of mapping this participant's recovery pattern was, therefore, to examine the relationship between spasticity management given two medications and neurobehavioral functioning.

Methods. Figure 1 illustrates the participant's neurobehavioral recovery pattern from time of first IP rehabilitation admission (42 days after injury) to time of discharge from her second IP rehabilitation stay (160 days after injury). The purpose of the second IP rehabilitation admission at 135 days after injury was to address unmanageable spasticity. At 135 days after injury (baseline), the participant's DOCS measure was 60 (Figure 1). She was not receiving any medications for spasticity, but she was receiving propranadol (20 mg TID), lansoprazole (15 mg QID), warfarin (5.5 mg BID), methylphenidate (10 mg QID), ibuprofen (400 mg BID), and enoxaparin (60 mg QID). Dantrolene (25 mg) was started 154 days after injury and increased to $50 \mathrm{mg}$ BID on 157 days after injury. The DOCS assessment was repeated after the dantrolene was therapeutic (157 days after injury), and a decline in neurobehavioral functioning from 60.44 to 43.64 was detected. Oral baclofen was started (10 mg QID) after completion of the DOCS evaluation 157 days after injury, and the dantrolene was stopped 158 days after injury. A DOCS assessment was repeated 160 days after injury; at which time, significantly improved neurobehavioral functioning was noted (from 44 to 57) after withdrawal of dantrolene but while still receiving oral baclofen (10 mg QID).

Results. Results indicate that while dantrolene improved spasticity management, dantrolene had an immediate effect of unintended sedation, which was sustained for the duration of time that the participant was on dantrolene. Spasticity improved at the cost of diminished neurobehavioral functioning. The attending physician opted to control spasticity with an intrathecal baclofen pump.

\section{Case 2: Short-Term Effects of Neurostimulants}

An 18-year-old male incurred a head injury subsequent to a motor vehicle accident in which he was an unbelted passenger in the back end of a flatbed truck. Prior to the administration of neuroparalytic agents, he presented with a GCS score of 3 in the emergency room where he underwent a left and right temporal resection secondary to edema. His acute hospitalization stay was complicated by extensive skull fractures with evidence of pneumocephali, increased intracranial pressure, bilateral decompressive craniotomy with duraplasty, C5 to T1 root avulsion, MRSA (Methicillin-Resistant Staphylococcus Aureus), reactive thrombosis, ventilatory support, tracheostomy, J-tube placement and inferior Vena Cava filter placement, and repair of right axillary artery tear. His IP rehabilitation stay was complicated by hyperadrenergic state and spasticity. He was admitted to IP rehabilitation 32 days after injury. He received 2.61 hours of therapy per IP rehabilitation day.

The family reported diminished activity while the participant was on methylphenidate. Chronologically, this participant is considered an adult, but given that the purpose of methylphenidate is to enhance inhibition in children, methylphenidate could plausibly be sedating to an 18-year-old with a severe BI. Therefore, we mapped this participant's recovery pattern so as to examine the short-term effects of a methylphenidate on neurobehavioral functioning for this participant.

Methods. The participant's neurobehavioral recovery pattern from time of first IP rehabilitation admission (32 days after injury) to 66 days after injury is illustrated 
in Figure 2. Baseline DOCS assessments were conducted 34 and 35 days after injury. At baseline, the participant was not receiving methylphenidate, but was receiving aspirin (325 mg BID), heparin (5,000 units QID), and lansoprazole (30 $\mathrm{mg}$ BID) at the time of IP rehabilitation admission and until discharge. Baseline DOCS measures were 54 and 53 DOCunits of functioning. The participant was started on $10 \mathrm{mg}$ QD of methylphenidate 36 days after injury and was titrated up to $20 \mathrm{mg}$ QD by 39 days after injury. The family and therapists reported reduced participation and responsiveness. The DOCS assessment was repeated 41 and 43 days after injury and corroborated reports of a decline in neurobehavioral functioning while methylphenidate was at a therapeutic level. Methylphenidate was withdrawn 44 days after injury, and the family and therapists reported improved participation and responsiveness 46 days after injury. A DOCS assessment completed 66 days after injury corroborated reports of improved neurobehavioral functioning (68 DOCunits) after withdrawal of methylphenidate.

Results. Methylphenidate 4 weeks after injury coincided with a reduction in the quality of neurobehavioral responses for this 18-year-old male participant. A DOCS evaluation was not able to be conducted immediately after medication withdrawal, but family and therapist reports indicated improved participation in therapy after medication withdrawal. The attending physician chose to not restart methylphenidate.

\section{Case 3: Determining Effective Seizure Control}

A 72-year-old female with a history of hypertension and diabetes mellitus with peripheral neuropathy incurred a severe BI when she fell on tiled flooring. She sustained a large right frontal hematoma. Her acute hospitalization stay was complicated by a subdural hematoma, hydrocephalus, and intraparenchymal hemorrhage for which she underwent craniotomy. She received seizure prophylaxis during 27 days of acute hospitalization (Dilantin, $300 \mathrm{mg}$ /day). The participant was admitted to IP rehabilitation directly from acute care, and she was discharged from IP rehabilitation 100 days after injury. IP rehabilitation stay was interrupted for 3 days for placement of a (VP) ventriculoperitoneal shunt. She received 2.2 hours of therapy per IP rehabilitation day.

Methods. A baseline DOCS evaluation was completed 30 days after injury (23 March) (Figure 3), and indicated 40 DOCunits of neurobehavioral functioning. At baseline, the participant's medications included heparin
(100 units/mL BID), enoxaparin (40 mg BID), epoetin alfa (40,000 units, BID), insulin glargine (units adjusted daily according to blood sugar levels), lidocaine (10 mL), sodium chloride (10 mL BID), phenytoin (i.e., Dilantin) (200 mg BID), Keppra (500 mg QID), metformin (500 mg BID), atenolol (50 mg BID), enalapril (10 mg BID), levothyroxine (0.025 mg BID), methylphenidate (5 mg BID), and amantadine (50 mg QID). All medications were continued until IP rehabilitation discharge, except lido-caine was stopped after one dose, enalapril was stopped on 24 March, amlodipine (5 mg BID) was stopped on 30 March, amantadine was stopped on 26 March, and methylphenidate was stopped on 1 April. Clonidine (0.1 mg BID) was started on 5 April titrated up to $0.2 \mathrm{mg}$ daily and continued throughout IP rehabilitation stay.

Therapists evaluated the participant with the DOCS every 7 days during IP rehabilitation, and the physician used weekly results as one mechanism of determining effective antiseizure medications and dose. After VP shunt placement, the participant had one episode of rightsided focal seizure activity on 12 April that lasted 5 minutes. Lorazepam (2 $\mathrm{mg} / \mathrm{mL}$ ) stopped the seizure activity after 5 minutes. After this episode, Keppra was increased to $1,500 \mathrm{mg}$ QID, but the DOCS neurobehavioral measure did not improve. An EEG was subsequently completed and indicated seizure activity that had not been observed. The physician then placed the participant on Trileptal (300 mg BID), and an EEG indicated no seizure activity. Her DOCS measure improved commensurately.

Results. Keppra coincided with continued decline in DOCS neurobehavioral measures serving as the catalyst for an EEG test that confirmed the presence of unobserved seizure activity. Trileptal coincided with an improved DOCS neurobehavioral measure. The attending physician chose to continue Trileptal at $300 \mathrm{mg}$ BID for the remainder of IP rehabilitation, and she was discharged on this medication and dosage schedule.

\section{Case 4: Development of Short- and Long-Term Rehabilitation Goals}

A 33-year-old male sustained a traumatic BI (TBI) in a motorcycle versus car accident in which the participant was the driver of the motorcycle. He wore no helmet. GCS before the administration of neuroparalytic agents was 5. A CT (computed tomography) scan revealed severe brain contusion with right subarachnoid hemorrhage and subdural hemorrhage with right shift. A left craniotomy with evacuation and intracranial pressure 
monitoring was completed. His acute care hospitalization was complicated by right lower-limb and left upper-limb deep vein thrombosis. During IP rehabilitation, the participant had increased lethargy and hydrocephalus was discovered on a CT scan. He was treated with placement of a VP shunt.

MethodsDuring the participant's IP rehabilitation, documentation of daily and weekly progress is essential to the ongoing refinement of individualized rehabilitation programs, and mapping this participant's recovery pattern guided this dynamic process. Weekly DOCS evaluations allowed the therapists to develop goals that (1) were measurable, (2) challenged the participant, and/or (3) were aligned with the participant's ability level. At baseline (116 days after injury), the participant received enoxaparin (60 mg QD), Diltiazem (30 mg TID), Amantadine (200 mg QID), hydralazine (25 mg QD), lansoprazole (30 mg BID), levetiracetam (250 mg BID), metropolol (100 mg QID), trazodone (200 mg BID), and warfarin (4 mg BID). Levetiracetam and enoxaparin were discontinued after 1 day. The participant also received sertraline (25 mg BID) 124 days after injury for 24 hours and $100 \mathrm{mg}$ BID 137 days after injury for 24 hours. Lansoprazole (30 mg BID) was also prescribed on day 137 for 24 hours. All other medications remained the same from baseline through 137 days after injury.

ResultsThe baseline DOCS evaluation yielded an overall neurobehavioral measure of 61 DOCunits. An examination of the participant's response pattern by modalities (Figure 4) indicated that higher-level localized responses were elicited with visual stimulation, whereas auditory, tactile, and taste stimulation elicited lower-level generalized responses. Therapists wrote initial goals to capitalize on the participant's strength and to use these strengths to build bridges toward the long-term goal of shaping and developing specific auditory responses such as following auditory commands. Shortterm goals included successfully eliciting higher-level localized auditory responses rather than establishing specific goals such as following a one-step command three times, which at this point would exceed the participant's ability. The weekly DOCS measures indicated overall improvement, especially in auditory and taste. As the patient exhibited an increased number of localized responses in these modalities, the therapists were able to adjust the patient's goals. The therapists were able to introduce command following, and they were able to establish a gestural yes-no response system that eventually led to a communication system in which visual forced choices of two objects were used to communicate basic needs.

\section{Neurobehavioral Mapping of Repeated DOCS Measures: Scientific Implementation}

In this section, we describe two intervention studies that illustrate how repeated DOCS measures are used or will be used in conjunction with fMRI to examine the relationship between changes in neural responses and changes in neurobehavioral functioning during coma recovery.

\section{Study 1}

Study 1 is a feasibility study and uses a single-subject design of experimental and control modalities to examine the effect of an intervention-familiar auditory stimulation. Each experimental subject is matched with a healthy control subject according to age, gender, and handedness. We are using fMRI because it is noninvasive and allows for good spatial resolution and assessment of interregional brain relationships, but it does not require explicit task performance. We are using DOCS because it allows for examination of the relationship between neural adaptation and behavioral changes. This is possible because test stimuli in DOCS have been designed to be compatible with fMRI.

We evaluate the experimental participant weekly with the DOCS and with fMRI at baseline and at completion of the intervention. The matched healthy control subject undergoes all the same research procedures as the experimental subject, except that the healthy control is not evaluated with the DOCS. Baseline tests are completed while participants are in the intensive care unit. An experimental intervention of listening to a familiar voice, in addition to standard rehabilitation, is subsequently started. The intervention is having the participant listen to a digital recording of a person known to the participant for at least 1 year prior to injury reading eight phonetically balanced paragraphs in randomized order. The participant listens to this 4-minute recording a minimum of three times a day for 21 days.

The within-subject experimental modality is auditory (i.e., auditory association area $=$ experimental), and the tactile modality (i.e., somatosensory area $=$ control) is the control modality. The fMRI assessments, therefore, include auditory and tactile sensory stimulation assessment paradigms. The auditory assessment paradigms 
have three conditions that are presented in a randomized order: the same familiar voice used for the intervention, a nonfamiliar gender-matched voice reading the same standard paragraphs, and rest. Using a digital sound-editing program, we standardize the familiar and nonfamiliar recordings according to amplitude. The order of presentation of the nonfamiliar and familiar recordings are then randomized and alternated with 30 seconds of rest (rest $=$ MRI noise) and subsequently burned onto a compact disc. The tactile assessment paradigm consists of alternating body temperature $\left(36^{\circ} \mathrm{C}\right)$, cold temperature (26 $\left.{ }^{\circ} \mathrm{C}\right)$, and hot $\left(42^{\circ} \mathrm{C}\right)$ temperature with the use of an fMRI-compatible stimuli delivery device.

The results will allow for an examination of whether daily exposure to a familiar voice improved auditory functioning neurobehaviorally and mechanistically. We will examine this by comparing the DOCS neurobehavioral auditory changes with tactile changes and by comparing fMRI auditory paradigm results with tactile paradigm results. We will also examine relationships between neurobehavioral changes by modality (i.e., auditory, tactile, visual, etc.) and neural adaptation. Then we will examine the results from the experimental group compared with the healthy control group to identify if neural recovery incrementally resembles neural activity in the healthy brain.

\section{Study 2}

Study 2 is a Phase I and II randomized clinical trial (sponsored by the Department of Veterans Affairs [VA], Rehabilitation Research and Development service, B3302K). This study started July 1, 2004, and requests for approvals from human subject IRBs and the Food and Drug Administration are, at the time of writing this paper, under review. The standard treatment of $20 \mathrm{mg}$ of methylphenidate daily for 6 weeks is being compared with repetitive transcranial magnetic stimulation daily for 6 weeks. These two treatments are provided in conjunction with standard rehabilitation. A third retrospective cohort serves as the standard rehabilitation control group. The indices of impairment are baseline and weekly DOCS and GCS neurobehavioral measures, fMRI, and quantitative EEG (QEEG). The fMRI design used in Study 1 just described is the same, but the auditory test stimuli are different and include stimuli that should elicit brain stem responses. After recovery of consciousness, the Galveston Orientation and Amnesia Test is the neurobehavioral index of impairment. Indices or outcomes are time to consciousness and recovery of functional skills. These measures are obtained at baseline and then weekly for the 6 weeks of treatment and again at 3,6, and 12 months after baseline.

The results from Study 2 will indicate whether methylphenidate and/or transcranial magnetic stimulation induces a state conducive to neural plasticity and whether each will facilitate neurobehavioral recovery with corresponding neural adaptation. The findings will also indicate whether one transcranial magnetic stimulation and/ or methylphenidate facilitates the recovery of consciousness and/or the recovery of functional skills.

\section{DISCUSSION}

Neurobehavioral recovery slopes enhance and augment medical and rehabilitation management because trends over time can quantitatively, reliably, and accurately define progress, plateaus, and/or declines. The evidence cited in the first paper (Part I found in this issue, page 1) and in this paper contributes to the body of data needed to develop evidence-based medical and rehabilitation management guidelines for persons recovering from coma. Weekly DOCS assessments of neurobehavioral functioning allowed the attending physician to determine that an intrathecal baclofen pump was the optimal treatment for spasticity management for Case 1. Weekly DOCS assessments for Case 2 allowed the treating physician to determine that responsiveness of his 18-year-old patient, contrary to clinical intuition, was diminished when receiving methylphenidate. Case 3 illustrated how routine weekly mapping of neurobehavioral recovery identified undetected seizure activity and the most effective medication for controlling the seizure activity. Case 4 illustrated how the rehabilitation team analyzed DOCS results globally and by modality at weekly conferences, which helped the team capitalize on each patient's strengths while maximizing weaknesses. This integrated interdisciplinary effort also set the stage for close alignment between rehabilitation and medical management. The ongoing and planned scientific investigations illustrate how science can be advanced given the capacity to reliably and accurately detect subtle changes in neurobehavioral functioning and illustrate the next step in science is to examine the relationships between behavior and neural adaptation.

It is important to elaborate on the clinical application of neurobehavioral recovery slopes and fMRI with severe 
TBI. Neurobehavioral recovery slopes should be defined with more than two time points. At least three time points should be used to signal/define a decline and/or an improvement. This action will facilitate interpretation of the trend/slope. A decline between two time points could be due, for example, to fatigue or reduced endurance rather than a secondary medical complication or pharmacological side effect. Furthermore, fMRI is very sensitive to changes in the physiological state (e.g., central nervous system stimulants). We must follow a rigorous research design to produce interpretable results (e.g., crossover designs) [19]. We must also use caution when interpreting improvements. A relationship between an intervention and improved recovery for a case study does not mean that it is a causal relationship. Alternative explanations could exist for the observed improvement.

Examination of individual recovery patterns with repeated DOCS measures can account for the impact of secondary brain damage, but modality specific analyses would be required. For example, a scientist can detect cortical blindness by examining person fit statistics and individual response profiles by modalities subsequently identifying modality-specific deficits. Person fit statistics can identify those persons who do not perform in a manner similar to the majority of the sample. Further inspection of that person's individual response profile can lead to additional information regarding secondary brain damage. If individual response patterns depart from the patterns predicted by the measurement model, then these will be identified as outliers (unexpected responses). If a person exhibits high scores on items in all modalities except visual, for example, then responses to visual items will not fit (misfit) with expected/predicted responses.

This final section is intended to illustrate the direction that science can take now that we have the capacity to examine the relationships between behavioral changes and neural adaptation. The capacity to directly compare behavioral changes with neural adaptation can advance rehabilitation research, but the clinical usefulness of QEEG and fMRI with unconscious persons is still under investigation. QEEG and fMRI with the severe TBI population are not routinely used clinically because the interpretability of the data remains unclear. These ongoing studies illustrate that the next scientific step is to examine the relationship between behavior and neural adaptation and that QEEG and fMRI might be tools that will allow for such an examination [20-21].

\section{CONCLUSIONS}

The case reports, together, suggest that a useful protocol for medical and rehabilitation management is to track individual recovery patterns during coma recovery. The systematic tracking of individual neurobehavioral recovery patterns, by clinicians with the DOCS, enhanced medical and rehabilitation management during coma recovery. The measurement of subtle and meaningful changes in neurobehavioral functioning-

1. Provided insights into the short-term effects of pharmacology.

2. Allowed for the detection of secondary medical complications.

3. Allowed the development and refinement of rehabilitation goals to be based on individualized evidence.

Rigorous studies examining treatment and rehabilitation effectiveness can be conducted, and in turn, a body of evidence guiding rehabilitation medicine can be assembled during coma recovery.

\section{ACKNOWLEDGMENTS}

We wish to acknowledge and thank the study participants and their family members. Their courage and strength to fear an inadequate life provide the light for our scientific journey. We also wish to recognize the contributions of the therapists at the Minneapolis VA Medical Center and the Rehabilitation Institute of Chicago, who assisted with data collection; to give a special thanks to Ms. Timilyn Williams, Bessie Weiss, and Ms. Judy Hill for their administrative and coordination efforts; and to send a note of thanks to Dr. Pape's VA career development comentors Drs. Frances Weaver and Allen Heinemann for their dedication, time, and carefully considered insights. We also appreciate the in-kind contributions from Marianjoy Rehabilitation Hospital.

\section{REFERENCES}

1. Practice parameters: Assessment and management of patients in the persistent vegetative state (summary statement). Report of the Quality Standards Subcommittee of the American Academy of Neurology. Neurology. 1995; 45(5):1015-18. 
2. Giacino JT, Zasler ND, Katz DI, Kelly JP, Rosenberg JH, Filley CM. Development of practice guidelines for assessment and management of the vegetative and minimally conscious states. J Head Trauma Rehabil. 1997;12(4):79-89.

3. Zafonte RD, Hammond FM, Peterson J. Predicting outcome in slow to respond traumatically brain-injured patients: Acute and subacute parameters. NeuroRehabilitation. 1996;6:19-32.

4. Zasler ND, Kreutzer JS, Taylor D. Coma stimulation and coma recovery: a critical review. NeuroRehabilitation. 1991; 1(3):33-40.

5. NIH Consensus Development Panel. Rehabilitation of persons with traumatic brain injury. JAMA. 1999;282(10): 974-83.

6. Andrews K. International Working Party Report on the Vegetative State: summary report. Brain Inj. 1996;10(11): 797-806.

7. Whyte J, Vaccaro M, Grieb-Neff P, Hart T. Psychostimulant use in the rehabilitation of individuals with traumatic brain injury. J Head Trauma Rehabil. 2002;17(4):284-99.

8. Zafonte RD, Cullen N, Lexell J. Serotonin agents in the treatment of acquired brain injury. J Head Trauma Rehabil. 2002;17(4):322-34.

9. Meythaler JM, Brunner RC, Johnson A, Novack TA. Amantadine to improve neurorecovery in traumatic brain injuryassociated diffuse axonal injury: a pilot double-blind randomized trial. J Head Trauma Rehabil. 2002;7(4):300-13.

10. Blount PJ, Nguyen CD, McDeavitt JT. Clinical use of cholinomimetic agents: a review. J Head Trauma Rehabil. 2002;17(4):314-21.

11. Lombardi F, Taricco M, De Tanti A, Telaro E, Liberati A. Sensory stimulation of brain-injured individuals in coma or vegetative state: results of a Cochrane systematic review. Clin Rehabil. 2002;16(5):464-72.
12. Whyte J, Glenn M. The care and rehabilitation of the patient in a persistent vegetative state. J Head Trauma Rehabil. 1986;1:39-53.

13. Giacino JT, Kalmar K. The vegetative and minimally conscious states: a comparison of clinical features and functional outcome. J Head Trauma Rehabil. 1997;12(4):36-51.

14. Giacino JT, Ashwal S, Childs N, Cranford R, Jennett B, Katz DI, Kelly JP, Rosenberg JH, Whyte J, Zafonte RD, Zasler ND. The minimally conscious state: definition and diagnostic criteria. Neurology. 2002;58(3):349-53.

15. Teasdale G, Jennett B. Assessment of coma and impaired consciousness: A practical scale. Lancet. 1974;2(7872): 81-84.

16. Medical aspects of the persistent vegetative state. Multisociety task force on PVS. N Engl J Med. 1994;330(21 Pt 1): 1499-1508 and 330(22 Pt 2):1572-79.

17. Plum F, Posner JB. The diagnosis of stupor and coma. 3d ed. Philadelphia: (PA): F.A. Davis Company; 1980.

18. Benjamin DW, Stone MH. Best test design. University of Chicago, Chicago (IL): MESA Press; 1979.

19. Whyte J, Hart T, Vaccaro M, Grieb-Neff P, Risser A, Polansky M, Coslett HB. Effects of methylphenidate on attention deficits after traumatic brain injury: a multidimensional, randomized, controlled trial. Am J Phys Med Rehabil. 2004;83(6):401-20.

20. Thatcher RW. QEEG and traumatic brain injury: Present and future. Brain Inj Source. 1999;3(4):20-22.

21. Pape T, Gitelman D, Parrish T, Senno R, Kelly JP, Weiner B. Measurement of cerebral activity in male participants four and six years post severe brain injury. Arch Phys Med Rehabil. 2001;82(9):1291-1346.

Submitted for publication March 8, 2004. Accepted in revised form August 2, 2004. 